\title{
Impaired Default Mode Network on Resting-State fMRI in Children with Medically Refractory Epilepsy
}

\author{
E. Widjaja, M. Zamyadi, C. Raybaud, O.C. Snead, and M.L. Smith
}

\begin{abstract}
BACKGROUND AND PURPOSE: Resting-state networks including the DMN have been shown to be abnormal in adults with temporal lobe epilepsy. However, little is known about the DMN in children with medically refractory epilepsy. The aim was to determine whether there was a difference in the DMN in children with medically refractory epilepsy relative to controls.
\end{abstract}

MATERIALS AND METHODS: Eleven children with medically refractory epilepsy and 11 age-matched healthy controls underwent restingstate fMRI. IC analysis was used to identify the DMN. A random-effects analysis was performed on the Z-maps of the DMN within each group and between groups. We calculated the temporal correlation coefficients of pairs of ROIs: PCC/PCUN, mPFC, and left and right lateral parietal cortices. The relations between $z$ scores of temporal correlation coefficients of pairs of ROIs and clinical seizure parameters and IQ were assessed.

RESULTS: The patients demonstrated decreased DMN connectivity in the PCC/PCUN, bilateral lateral parietal cortex, and anterior and midcingulate relative to controls. There was reduced connectivity between the mPFC-right lateral parietal cortex, the PCC/PCUN-left lateral parietal cortex, and the PCC/PCUN-right lateral parietal cortex pairs of ROIs in patients compared with controls. There were no significant correlations between the $z$ scores of temporal correlation coefficients of the 6 pairs of ROls in patients and age of seizure onset, duration of epilepsy, number of medications, seizure frequency, and IQ.

CONCLUSIONS: We have found reduced connectivity in the DMN in children with medically refractory epilepsy. Further studies are needed to determine whether different seizure types have different effects on the DMN and whether the impaired connectivity is related to cognitive functions subserved by the DMN.

ABBREVIATIONS: DMN = default mode network; $\mathrm{EEG}=$ electroencephalogram; IC = independent component; IQ = intelligence quotient; $\mathrm{mPFC}=$ medial prefrontal cortex; $\mathrm{PCC} / \mathrm{PCUN}=$ posterior cingulate cortex/precuneus

$F^{\prime}$ unctional connectivity is defined as the temporal synchronization of low-frequency fluctuations $(0.01-0.1 \mathrm{~Hz})$ arising from spontaneous neuronal activities in distant brain regions. ${ }^{1}$ The spontaneous fluctuations are measured at rest, the method is referred to as "resting-state" fMRI, and the identified brain networks are referred to as resting-state networks. The DMN, which is one of the resting-state networks, is a prominent large-scale brain network that includes the PCC/PCUN, mPFC, lateral parietal cortex, and medial temporal lobes. The DMN has high resting

Received May 4, 2012; accepted after revision June 7.

From the Department of Diagnostic Imaging (E.W., M.Z., C.R.), Division of Neurology (O.C.S.), Hospital for Sick Children, and Department of Psychology (M.L.S.), University of Toronto, Toronto, Ontario, Canada.

Please address correspondence to E. Widjaja, MD, Diagnostic Imaging, Hospital for Sick Children, 555 University Ave, Toronto, Ontario M5G 1X8, Canada; e-mail: Elysa.Widjaja@sickkids.ca

Evidence-Based Medicine Level 2.

http://dx.doi.org/10.3174/ajnr.A3265 metabolism, deactivates during cognitively demanding tasks, ${ }^{2}$ and demonstrates increased activity during high-level social cognitive tasks. ${ }^{3}$ Individual brain regions that make up the DMN are involved in the integration of autobiographic, self-monitoring, and social cognitive functions. ${ }^{4}$ The PCC/PCUN is activated during tasks that involve autobiographic memory and self-referential processes. ${ }^{5}$ The $\mathrm{mPFC}$ is associated with social cognitive processes related to self and others. ${ }^{6}$ The medial temporal lobes are engaged in episodic memory, ${ }^{7}$ and the lateral parietal cortex is involved in spatial attention. ${ }^{8}$ However, the precise functions collectively subserved by the DMN are still largely unknown.

Prior studies have evaluated a variety of resting-state networks in adults with mesial temporal lobe epilepsy ${ }^{9-13}$ and generalized tonic clonic seizures. ${ }^{14}$ Gotman et $\mathrm{al}^{15}$ found that spike and wave discharges in epilepsy were associated with deactivations in the default state of the brain. This deactivation of the resting-state network by epileptiform discharges may be associated with impaired cognitive function in patients with epilepsy. Zhang et $\mathrm{al}^{12}$ 
Table 1: Clinical characteristics of patients

\begin{tabular}{|c|c|c|c|c|c|}
\hline $\begin{array}{l}\text { No./Age } \\
\text { (yr)/Sex }\end{array}$ & Location of Epilepsy & $\begin{array}{c}\text { Age at Seizure } \\
\text { Onset (yr) }\end{array}$ & $\begin{array}{l}\text { Duration of } \\
\text { Epilepsy (yr) }\end{array}$ & Antiepileptic Medications & $\begin{array}{c}\text { Seizure } \\
\text { Frequency/Week }\end{array}$ \\
\hline $1 / 15.2 / F$ & R frontal & 7 & 8 & Lamotrigine, levetiracetam & 7 \\
\hline $2 / 16.3 / F$ & $\mathrm{R}$ frontal paracentral & 14 & 2.3 & Carbamazepine & 7 \\
\hline $3 / 10.0 / F$ & R neocortical inferior temporal & 7 & 3 & Lamotrigine, topiramate & 10 \\
\hline 4/15.3/F & $\mathrm{R}$ frontal paracentral & 3 & 12.3 & Carbamazepine, lamotrigine, divalproex & 10 \\
\hline $5 / 14.0 / \mathrm{M}$ & L frontal & 8 & 6 & Valproic acid, lamotrigine & 20 \\
\hline $6 / 10.1 / F$ & L neocortical anterior temporal & 8 & 2 & Carbamazepine & 10 \\
\hline $7 / 16.2 / \mathrm{M}$ & L frontal paracentral & 8 & 8 & Carbamazepine, levetiracetam & 10 \\
\hline $8 / 14.1 / \mathrm{M}$ & L neocortical anterior temporal & 12 & 2 & Lamotrigine, levetiracetam & 2 \\
\hline $9 / 14.7 / F$ & $\mathrm{R}$ hemisphere & 13 & 1 & Levetiracetam, divalproex, valproic acid & 2.5 \\
\hline $10 / 15.0 / F$ & L frontal & 4.5 & 10.5 & Oxcarbazepine & 40 \\
\hline $11 / 14.1 / F$ & $\mathrm{R}$ lateral parietal occipital & 10 & 4 & Levetiracetam, topiramate & 40 \\
\hline
\end{tabular}

Note:- $R$ indicates right; L, left.

previously showed that patients with mesial temporal lobe epilepsy have decreased functional connectivity in almost all the regions within the dorsal attention network. This reduction in functional connectivity within the dorsal attention network was negatively correlated with the Trail-Making Test score, a test of attention function. To date most studies investigating restingstate networks in patients with epilepsy have focused on adults with long-standing epilepsy. It is not known whether children with epilepsy also have impairment in the resting-state network and whether there is a relation between DMN and IQ, because reduced IQ is common in children with medically refractory epilepsy ${ }^{16,17}$ and IQ provides a global measure of cognitive function.

We hypothesized that ictal and/or interictal activity in children with medically refractory epilepsy leads to reduced functional connectivity within the DMN. The secondary hypothesis is that there is an association between impaired DMN and longer duration of epilepsy and impaired IQ. The aim of this study was to determine whether there is a difference in the DMN as measured by using resting-state fMRI in children with poorly controlled epilepsy relative to controls. The secondary aim was to determine whether there is an association between impaired DMN and clinical measures of seizure severity and IQ.

\section{MATERIALS AND METHODS \\ Subjects}

The study was approved by the local institutional research ethics board. Eleven children, mean age of $14.1 \pm 2.1$ years (range, 10.016.3 years), consisting of 6 girls and 5 boys with medically refractory epilepsy who were being evaluated for epilepsy surgery were recruited (Table 1). The mean age of seizure onset was $8.6 \pm 3.4$ years, the mean duration of epilepsy was $5.4 \pm 3.8$ years, and the mean seizure frequency was 14.4 per week (range, 2-40). The mean number of medications in patients was 1.9 (range, 1-3). Seven patients had complex partial seizures, 1 had complex partial seizures with secondary generalization, 2 had simple partial seizures, and 1 had simple partial seizures with secondary generalization. Video EEG and seizure semiology localized the seizure to the frontal lobe in 6 patients (including 3 in the paracentral region), the neocortical temporal lobe in 3 patients, the right lateral parietal-occipital lobe in 1 patient, and the right hemisphere in 1 patient. All the children with epilepsy had normal MR imaging findings and were thus nonlesional. Eleven age-matched healthy control subjects consisting of 6 girls and 5 boys with normal MR imaging findings and no neurologic or psychiatric disorders were included. The mean age of the controls was $14.5 \pm 2.4$ years (range, 10.7-16.9 years). There was no significant difference between the ages of the patients and controls $(P>.05)$.

\section{MR Imaging}

MR imaging was performed on a 3T scanner (Achieva magnet; Philips Healthcare, Best, the Netherlands) by using an 8-channel phased array head coil in all patients and controls. Patients were imaged by using the epilepsy protocol, which included axial and coronal FLAIR $(\mathrm{TR} / \mathrm{TE}=10,000 / 140 \mathrm{~ms}$, section thickness $=3$ $\mathrm{mm}, \mathrm{FOV}=22 \mathrm{~cm}$, matrix $=316 \times 290)$, axial and coronal T2 and proton attenuation $(\mathrm{TR} / \mathrm{TE}=4200 / 80 / 40 \mathrm{~ms}$, section thickness $=3 \mathrm{~mm}, \mathrm{FOV}=22 \mathrm{~cm}$, matrix $=400 \times 272)$, volumetric $3 \mathrm{D}$ $\mathrm{T} 1(\mathrm{TR} / \mathrm{TE}=4.9 / 2.3 \mathrm{~ms}$, section thickness $=1 \mathrm{~mm}, \mathrm{FOV}=22$ $\mathrm{cm}$, matrix $=220 \times 220)$, and resting-state $\mathrm{fMRI}(\mathrm{TR}=2000 \mathrm{~ms}$, $\mathrm{TE}=30 \mathrm{~ms}$, flip angle $=90^{\circ}, \mathrm{FOV}=220 \mathrm{~mm}$, matrix $=88 \times 86$ $\mathrm{mm}$, section thickness $=5 \mathrm{~mm}, 180$ volumes acquired and aligned to the anterior/posterior commissure line). Resting-state fMRI was performed with the patient's eyes closed. The imaging in controls included axial FLAIR, T2 and proton attenuation, volumetric 3D T1, and resting-state fMRI, which were performed with the same parameters as those in patients. The 3T MRI of patients and controls were reviewed by a pediatric neuroradiologist (E.W.) with expertise in epilepsy imaging, to verify that there was no lesion seen on MR imaging.

\section{Data Preprocessing}

A series of data-preprocessing steps were carried out by using both AFNI (http://afni.nimh.nih.gov/) ${ }^{18}$ and FSL (http://www. fmrib.ox.ac.uk/fsl $/)^{19}$ software packages. The details of the preprocessing steps are as follows: To allow T1 equilibrium effects, we discarded the first 10 volumes of each fMRI run. All volumes of the fMRI dataset were rigidly registered to the first volume of the same run (3dvolreg; AFNI). Section-timing correction was then performed on the motion-corrected data (3dTshift; AFNI). The differences in translational and rotational head motion between the patient and control groups were assessed. ${ }^{20}$ There were no significant differences for translational motion $(P=.4995)$ and rotational motion $(P=.2271)$ between the 2 groups. Next, both the anatomic T1 and the fMRI datasets were skull-stripped by using AFNI. To achieve atlas registration, we aligned the fMRI image to the high-resolution anatomic $\mathrm{T} 1$ image by computing an 
affine transformation (Linear Image Registration Tool of fMRI of the Brain; FSL). The T1 image was then affine-registered to MNI152 brain template of the Montreal Neurological Institute. The 3 transformations were then concatenated and used to resample the fMRI image to the MNI atlas space with a 2-mm isotropic voxel resolution. The fMRI data were then spatially smoothed by using a Gaussian kernel of $8-\mathrm{mm}$ full width at half maximum.

\section{Independent Component Analysis}

To identify the DMN, we used spatial IC analysis to decompose the data of all individuals in both groups into 25 ICs. IC analysis aims to identify temporally coherent networks by estimating maximally independent spatial sources. ${ }^{21}$ Each component is associated with a waveform that corresponds to the temporal profile of a specific pattern of coherent brain activity and a spatial map that expresses the intensity with which this connectivity is found across the voxels. ${ }^{22}$ We used the Infomax algorithm implemented in the group IC analysis of the fMRI Toolbox (GIFT) (http://mialab.mrn.org/software/gift/, Version 2.0e). ${ }^{23}$ The number of compo-

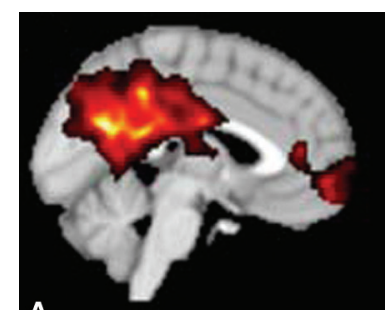

A
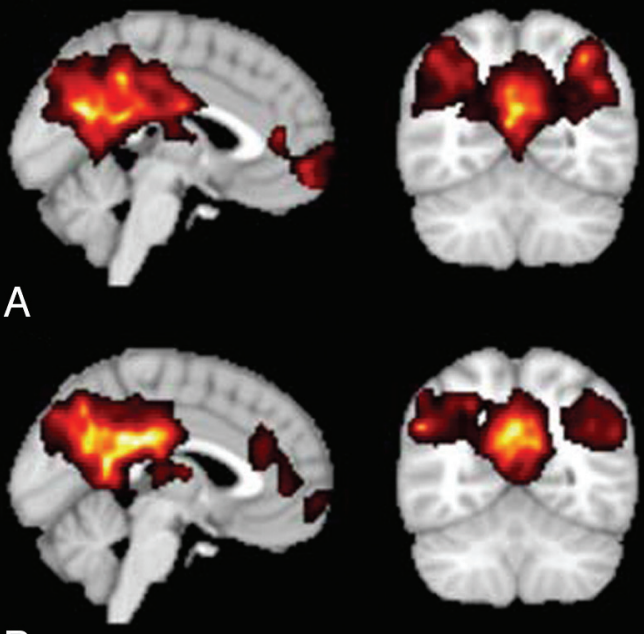

B

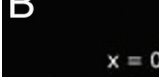

$x=0$

$y=-63$

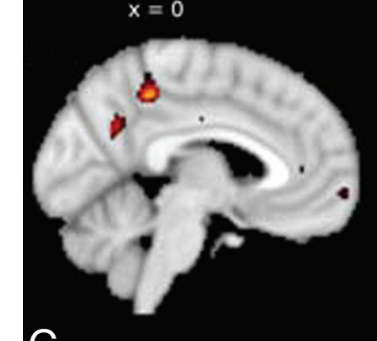

C $x=-7$

$y=-58$

FIG 1. Random-effects analysis of the DMN in controls $(A)$ and $(B)$ patients. $C$, The patients demonstrate decreased DMN connectivity in the PCC/PCUN, bilateral lateral parietal cortex, and anterior and midcingulate. nents for each individual subject was esti-

mated by using the minimum description length criterion ${ }^{24}$ built into GIFT. The average of individual IC numbers (ie, 25) was then used for all the individuals.

All ICs were spatially correlated with a DMN template (within the GIFT toolbox). The DMN template included the bilateral lateral parietal cortex, the mPFC, PCC/PCUN, and medial temporal lobes. ${ }^{25}$ The component corresponding to the DMN was then extracted from all subjects in both groups. Intensity values in each spatial DMN map were converted to $z$ scores to reflect the degree to which the time-series of each voxel correlates with the timeseries of the DMN component, scaled by the SD of the error term. ${ }^{23}$ In other words, the $z$ score is a measure of how many SDs the signal is from the background noise. ${ }^{26}$

\section{Within- and Between-Group Analysis of the DMN}

A random-effects analysis was performed on the Z-maps of the DMN within each group by using a 1-sample $t$ test. Thresholds were set at $P<.05$, after correction for multiple comparisons by using the false discovery rate. Next, a 2-sample $t$ test was performed to compare the $Z$-maps of the DMN between the patient and control groups. In this case, the threshold was set at $P<.05$, and a minimum cluster size of 15 voxels was used. The group comparison was masked to the voxels within the networks of either the control or patient groups (as defined by $t$ test, $P<.05$, false discovery rate-corrected). ${ }^{10,27}$

\section{Functional Connectivity within the DMN}

Four ROIs in the PCC/PCUN, mPFC, and left/right lateral parietal cortices were selected from the false discovery rate-corrected DMN map of the control group. The same ROIs were used in both the patient and control groups. The following preprocessing steps were performed on each region of interest: First, the average time-series was extracted from each region of interest. Then, linear regression analysis was used to remove several sources of spurious variance, which were unlikely to represent regionally specific correlations of neuronal origin. The nuisance variables included the 6 parameters obtained from rigid-body head-motion correction, and the signal was averaged over the time-series of $3 \mathrm{CSF}$ and 6 white matter ROIs located in different regions of the brain. ${ }^{28,29}$ All these signals were regressed out simultaneously by using the $3 \mathrm{dDeconvolve} \mathrm{program}$ in AFNI. The residual average time-series after regression was used for further pair-wise correlation analyses. ${ }^{13}$ To quantify the functional connectivity within the DMN, we used the Pearson correlation to calculate temporal correlation coefficients between all 6 pairs of ROIs.

\section{Correlation between Connectivity in the DMN and Clinical Parameters}

The relations between $z$ scores of the temporal correlation coefficients between 6 pairs of ROIs and clinical seizure parameters, including age at seizure onset, duration of epilepsy, number of med- 
Table 2: Location of brain regions in the default mode network of the controls ${ }^{\mathrm{a}}$

\begin{tabular}{lclc}
\hline Anatomic Regions & Hemisphere & $\begin{array}{c}\text { MNI } \\
\text { Coordinates } \\
(\mathbf{x}, \mathbf{y}, \mathbf{z})\end{array}$ & $\begin{array}{c}\text { Peak } \\
\boldsymbol{T} \text { Value }\end{array}$ \\
\hline $\begin{array}{c}\text { Posterior cingulate cortex/ } \\
\text { precuneus }\end{array}$ & $\mathrm{L} / \mathrm{R}$ & $-6,-54,28$ & 15.87 \\
Medial prefrontal cortex & $\mathrm{L} / \mathrm{R}$ & $-4,58,-10$ & 13.89 \\
Lateral parietal cortex & $\mathrm{L}$ & $-44,-62,33$ & 9.91 \\
& $\mathrm{R}$ & $42,-63,33$ & 10.51 \\
\hline
\end{tabular}

Note:- $R$ indicates right; L, left.

${ }^{a} P<.05$ after correction for multiple comparison using false discovery rate.
Table 3: Regions demonstrating reduced connectivity in patients relative to controls

\begin{tabular}{llcc}
\hline \multicolumn{1}{c}{ Anatomic Regions } & $\begin{array}{c}\text { MNI } \\
\text { Coordinates } \\
(\mathbf{x}, \mathbf{y}, \text { and } \mathbf{z})\end{array}$ & $\begin{array}{c}\text { Voxel } \\
\text { Size } \\
\left(\mathbf{m m}^{3}\right)\end{array}$ & $\begin{array}{c}\text { Peak } \\
\mathbf{T} \text { Value }\end{array}$ \\
\hline Right anterior cingulate & $4,40,8$ & 75 & 3.549 \\
$\begin{array}{l}\text { Right midcingulate } \\
\text { Right posterior cingulate cortex/ }\end{array}$ & $4,-16,40$ & 55 & 2.679 \\
$\quad 10,-68,52$ & 23 & 2.214 \\
$\begin{array}{l}\text { Right lateral parietal cortex } \\
\text { Left lateral parietal cortex }\end{array}$ & $42,-52,56$ & 183 & 3.606 \\
$\begin{array}{l}\text { Left posterior cingulate cortex/ } \\
\text { precuneus }\end{array}$ & $0,-60,34$ & 91 & 2.682 \\
\hline
\end{tabular}

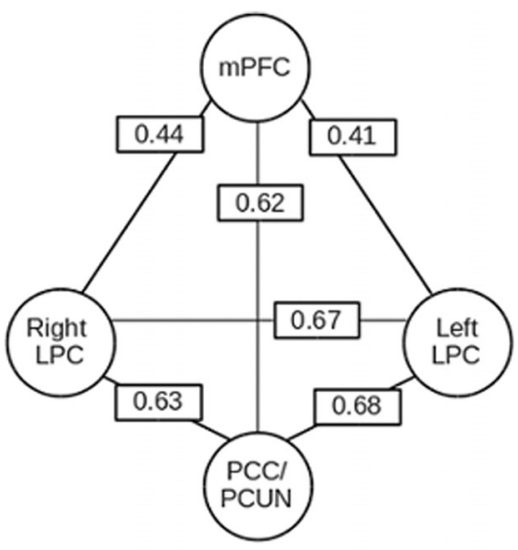

A

Controls

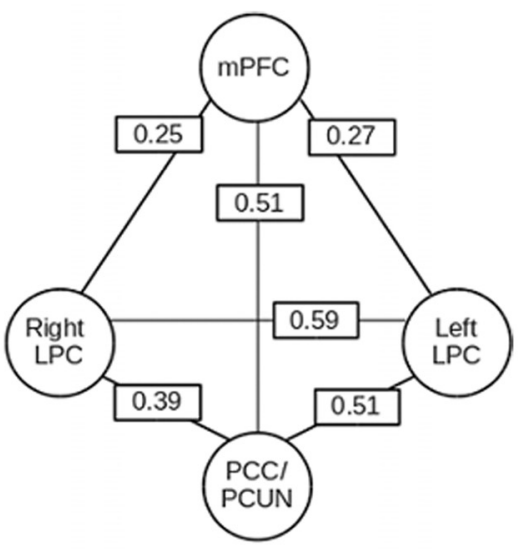

B

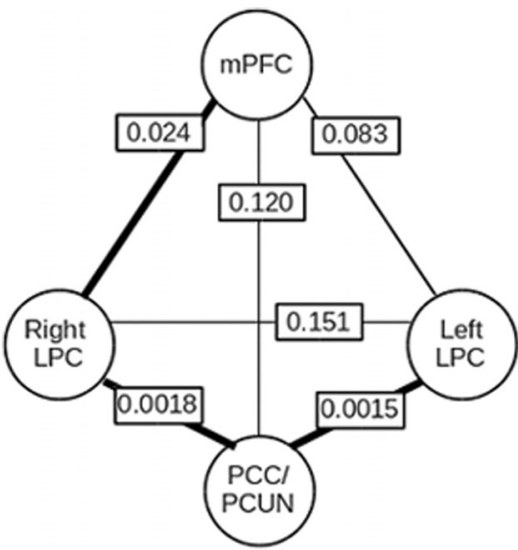

Controls vs. Patients

FIG 2. Functional connectivity within the $D M N$ in controls $(A)$ and patients $(B)$. Numbers in the rectangles correspond to the average pair-wise correlation coefficients. C, Comparison of functional connectivity within the DMN in controls versus patients shows significant reduction in mPFC-right lateral parietal cortex (LPC), PCC/PCUN-right LPC, and PCC/PCUN-left LPC. Numbers in rectangles represent the $P$ value for the comparison of the mean correlation coefficients of control and patient groups. Thick lines represent significant changes in the pair-wise correlation between controls and patients $(P<.05)$.

ications, seizure frequency, and IQ, were assessed by using the Pearson correlation.

\section{RESULTS}

\section{Functional Connectivity of the DMN in Each Group}

The random-effects analyses of IC maps in the patient and control groups showed the spatial pattern of the DMN $(P<.05$ after false discovery rate) (Fig 1). The DMN pattern in the controls demonstrated functional connectivity among the PCC/PCUN, mPFC, and bilateral lateral parietal cortex (Fig $1 A$ and Table 2). The DMN pattern of the patients showed similar regions of functional connectivity, as shown in Fig $1 B$. The patients demonstrated decreased DMN connectivity in the PCC/PCUN, bilateral lateral parietal cortex, and anterior and midcingulate (Fig $1 C$ and Table 3).

\section{Functional Connectivity of the DMN between Patients and Controls}

The $z$ scores of the 6 pairs of functional connectivity in patients and controls are shown in Fig 2. There was reduced connectivity between the following pairs of ROIs in patients compared with controls: the mPFC-right lateral parietal cortex $(P=.0252)$, the PCC/PCUN-left lateral parietal cortex $(P=.008)$, and the PCC/ PCUN-right lateral parietal cortex $(P=.0101)$. There were no significant differences between the following pairs of ROIs in pa- tients relative to controls: right lateral parietal cortex-left lateral parietal cortex $(P=.0756), \mathrm{mPFC}-$ left lateral parietal cortex $(P=$ $.0931)$, and PCC/PCUN-mPFC $(P=.0984)$. None of the pairs of regions of interest demonstrated increased connectivity in patients relative to controls.

\section{Correlation between Connectivity in the DMN and Clinical Parameters}

There were no significant correlations between the $z$ scores of the temporal correlation coefficients of the 6 pairs of ROIs in patients and age of seizure onset $(P>.05$ for all), duration of epilepsy $(P>$ $.05)$, number of medications $(P>.05)$, and seizure frequency $(P>.05)$. IQ was lower in patients (mean, $98.5 \pm 14.8)$ than in controls (mean, $110.0 \pm 9.8)(P=.043)$. No significant correlations were detected between the $z$ scores of the temporal coefficient of the 6 pairs of ROIs in patients and IQ $(P>.05$ for all).

\section{DISCUSSION}

Using resting-state fMRI, we have found impaired connectivity within the DMN in children with poorly controlled epilepsy, with reduced connectivity between the $\mathrm{mPFC}$-right lateral parietal cortex, PCC/PCUN-left lateral parietal cortex, and the PCC/ PCUN-right lateral parietal cortex. This reduction in functional connectivity may be related to a variety of factors, including ictal 
and interictal epileptiform discharges, ${ }^{30}$ frequent spread of seizure activities, and malformations of cortical development, that are not apparent on MR imaging. We have used IC analysis to retrieve patterns of coherent functional connectivity across all voxels in the brain, which permits evaluation of global connectivity. IC analysis has the advantage of not having to specify a priori the anatomic ROIs of the network.

Previous studies have reported a reduction in functional connectivity in the resting-state networks ${ }^{13}$ or a combination of reduced and increased connectivity in the resting-state networks in adults with epilepsy. ${ }^{9,14}$ Wang et al $^{14}$ previously evaluated the resting-state networks in patients with generalized tonic clonic seizures and have found regions of decreased as well as increased functional connectivity in the DMN and dorsal attention networks. Bettus et $\mathrm{al}^{9}$ evaluated the basal functional connectivity in adults with medial temporal lobe epilepsy and found that in those with left medial temporal lobe epilepsy, there was a trend toward decreased connectivity of the left anterior and posterior hippocampi link and a trend toward increased connectivity of the right anterior and posterior hippocampi link. Mankinen et $\mathrm{al}^{31}$ evaluated the resting-state connectivity of children with nonlesional temporal lobe epilepsy. They found reduced connectivity in the anterior component of the DMN, visual dorsal cortex, and pontine and somatosensory cortex resting-state network.

Subgroup analysis of patients with temporal lobe epilepsy and abnormal EEG findings showed reduced connectivity in 4 ICs and increased connectivity in 1 component, while those with temporal lobe epilepsy and normal EEG findings had reduced connectivity in 3 ICs. The reduction in functional connectivity is thought to reflect a dysfunction in the network, while an increase in functional connectivity is thought to reflect a compensatory mechanism or reorganization of the network. We did not find an increase in the functional connectivity in the DMN, a result similar to that reported in the study by Liao et al. ${ }^{13}$ It is possible that some seizure types or functional networks or certain epileptogenic substrates such as hippocampal sclerosis may be more likely associated with compensatory increased connectivity than others or that there is a period of time with epilepsy that is necessary before the compensation is observed. Further work is needed to clarify which patient populations, functional networks, or epileptogenic substrates are more likely to be associated with compensatory increased connectivity and to determine the clinical relevance of this compensatory connectivity.

We have not found a correlation between the strength of connectivity in the DMN and clinical parameters such as age at seizure onset, duration of epilepsy, number of medications, and seizure frequency. Wang et $\mathrm{al}^{14}$ found a negative correlation between seizure duration and functional connectivity in the medial prefrontal cortex in patients with generalized tonic clonic seizures. Zhang et $\mathrm{al}^{10}$ also found a negative correlation between epilepsy duration and the mean $z$ values within the regions that showed reduced functional connectivity in the visual, auditory, and sensorimotor networks in patients with temporal lobe epilepsy. The difference in the findings between the reported studies and our findings could be related to differences in the duration of epilepsy. The mean duration of epilepsy in those studies in adults varied from 10.6 to 13.0 years, ${ }^{10,14}$ which was at least twice as long as the mean duration of epilepsy in our patients. Longer duration of epilepsy may lead to greater impairment in resting-state networks. Alternatively, the more homogeneous group of patients with a specific type of epilepsy in those studies ${ }^{10,14}$ may have contributed to the observed association between epilepsy duration and resting-state functional connectivity. The lack of correlation between the strength of the connectivity in the DMN and clinical parameters in our study could also be related to the relatively small sample size and variations in seizure types. We did not identify an association between the number of antiepileptic medications and impaired DMN. However, this does not preclude the possibility of specific antiepileptic medications exerting a negative influence on resting-state connectivity.

We have not identified the medial temporal lobe component of the DMN within the patient and control groups. Because the medial temporal lobe is located close to the skull base and sphenoid sinus, it is possible that gradient artifacts reduce the signal from the medial temporal lobe. Failure to identify the medial temporal lobe is unlikely due to immaturity of the DMN in children. Supekar et $\mathrm{al}^{32}$ found that the DMN, including the medial temporal lobe component, could be identified in children 7-9 years of age and that the connectivity and strength of the medial temporal lobe was similar in children and adults. One of the potential limitations of this study is the small number of patients with a wide variety of partial seizures. Despite the variations in seizure types, we found reduced connectivity in the DMN. Impairment in the DMN does not appear to be specific to the subtypes of seizures and has been described in temporal lobe epilepsy, ${ }^{13}$ generalized tonic clonic seizures, ${ }^{14}$ and absence of epilepsy. ${ }^{33}$ Certain types of epilepsy, such as mesial temporal lobe epilepsy, could have a direct influence on the DMN because the seizure focus included the medial temporal lobe component of the DMN.

In our study, in some patients such as case 11, the seizure focus in the right lateral parietal occipital lobe included the lateral parietal cortex component of the DMN, but in others, such as those with seizures arising from the neocortical temporal lobe (cases 3 , 6 , and 8 ) or paracentral region (cases 2,4 , and 7), the seizure focus did not include the DMN. Hence, other factors, such as frequent spread of seizure activities, may have contributed to the reduced connectivity in the DMN, in addition to the epileptiform discharges within the seizure focus. It is not known whether patients with generalized epilepsy are more likely to have greater impairment in some resting-state networks such as the DMN relative to patients with partial epilepsy and whether those with partial epilepsy and secondary generalization are more likely to have greater impairment in resting-state networks than those with partial epilepsy but without secondary generalization. Due to the small number of patients with partial epilepsy and secondary generalization, we have not performed subgroup analysis. Another limitation of this study was that we have not assessed those cognitive functions subserved by the DMN, such as autobiographic memory, episodic memory, and social cognitive function. We have assessed the IQs of patients and controls and have found no correlation between impaired IQ and reduced functional connectivity. Although IQ provides a global measure of cognitive function, it is not directly related to DMN. 


\section{CONCLUSIONS}

We have found reduced connectivity in the DMN in children with medically refractory epilepsy. Further study is needed to determine whether different types of seizures have different effects on the strength of connectivity within the DMN. In addition, simultaneous recording of EEG during the DMN determination would allow us to determine whether interictal spikes and spike-wave activity on the EEG correlate with alteration of the DMN. We have not found an association between reduced connectivity in the DMN and reduced IQ. However, correlation study between DMN and cognitive functions subserved by the network, such as autobiographic memory, episodic memory, and attention, is needed to further understand the significance of the impaired connectivity in the DMN.

Disclosures: Elysa Widjaja—UNRELATED: Grants/Grants Pending: Derek HarwoodNash Chair, * GE-Association of University Radiologists award, * SickKids Foundation/ Institute for Human Development, Child and Youth Health-Center for Health Information and Research.* *Money paid to the institution.

\section{REFERENCES}

1. Fox MD, Snyder AZ, Vincent JL, et al. The human brain is intrinsically organized into dynamic, anticorrelated functional networks. Proc Natl Acad Sci U S A 2005;102:9673-78

2. Raichle ME, MacLeod AM, Snyder AZ, et al. A default mode of brain function. Proc Natl Acad Sci U S A 2001;98:676-82

3. Harrison BJ, Pujol J, Lopez-Sola M, et al. Consistency and functional specialization in the default mode brain network. Proc Natl Acad Sci U S A 2008;105:9781-86

4. Spreng RN, Mar RA, Kim AS. The common neural basis of autobiographical memory, prospection, navigation, theory of mind, and the default mode: a quantitative meta-analysis. $J$ Cogn Neurosci 2009;21:489-510

5. Buckner RL, Carroll DC. Self-projection and the brain. Trends Cogn Sci 2007;11:49-57

6. Amodio DM, Frith CD. Meeting of minds: the medial frontal cortex and social cognition. Nat Rev Neurosci 2006;7:268-77

7. Milner B. The medial temporal-lobe amnesic syndrome. Psychiatr Clin North Am 2005;28:599-611, 609

8. Chambers CD, Payne JM, Stokes MG, et al. Fast and slow parietal pathways mediate spatial attention. Nat Neurosci 2004;7:217-18

9. Bettus G, Guedj E, Joyeux F, et al. Decreased basal fMRI functional connectivity in epileptogenic networks and contralateral compensatory mechanisms. Hum Brain Mapp 2009;30:1580-91

10. Zhang Z, Lu G, Zhong Y, et al. Impaired perceptual networks in temporal lobe epilepsy revealed by resting fMRI. J Neurol 2009;256: 1705-13

11. Waites AB, Briellmann RS, Saling MM, et al. Functional connectivity networks are disrupted in left temporal lobe epilepsy. Ann Neurol 2006;59:335-43

12. Zhang Z, Lu G, Zhong $Y$, et al. Impaired attention network in temporal lobe epilepsy: a resting FMRI study. Neurosci Lett 2009;458: 97-101

13. Liao W, Zhang Z, Pan Z, et al. Default mode network abnormalities in mesial temporal lobe epilepsy: a study combining fMRI and DTI. Hum Brain Mapp 2011;32:883-95

14. Wang Z, Lu G, Zhang Z, et al. Altered resting state networks in epileptic patients with generalized tonic-clonic seizures. Brain Res 2011;1374:134-41

15. Gotman J, Grova C, Bagshaw A, et al. Generalized epileptic discharges show thalamocortical activation and suspension of the default state of the brain. Proc Natl Acad Sci U S A 2005;102:15236-40

16. D’Argenzio L, Colonnelli MC, Harrison S, et al. Cognitive outcome after extratemporal epilepsy surgery in childhood. Epilepsia 2011; 52:1966-72

17. Souza-Oliveira C, Escosi-Rosset S, Funayama SS, et al. Intellectual functioning in pediatric patients with epilepsy: a comparison of medically controlled, medically uncontrolled and surgically controlled children [Article in English, Portuguese]. J Pediatr (Rio J) 2010;86:377-83

18. Cox RW. AFNI: software for analysis and visualization of functional magnetic resonance neuroimages. Comput Biomed Res 1996; 29:162-73

19. Smith SM, Jenkinson M, Woolrich MW, et al. Advances in functional and structural MR image analysis and implementation as FSL. Neuroimage 2004;23(suppl 1):S208-19

20. Liu Y, Liang M, Zhou Y, et al. Disrupted small-world networks in schizophrenia. Brain 2008;131:945-61

21. Calhoun VD, Adali T, Pearlson GD, et al. Spatial and temporal independent component analysis of functional MRI data containing a pair of task-related waveforms. Hum Brain Mapp 2001;13:43-53

22. Celone KA, Calhoun VD, Dickerson BC, et al. Alterations in memory networks in mild cognitive impairment and Alzheimer's disease: an independent component analysis. J Neurosci 2006;26:10222-31

23. Calhoun VD, Adali T, Pearlson GD et al. A method for making group inferences from functional MRI data using independent component analysis. Hum Brain Mapp 2001;14:140-51

24. Li YO, Adali T, Calhoun VD. Estimating the number of independent components for functional magnetic resonance imaging data. Hum Brain Mapp 2007;28:1251-66

25. Greicius MD, Srivastava G, Reiss AL, et al. Default-mode network activity distinguishes Alzheimer's disease from healthy aging: evidence from functional MRI. Proc Natl Acad Sci U S A 2004;101: 4637-42

26. Greicius MD, Kiviniemi V, Tervonen O, et al. Persistent defaultmode network connectivity during light sedation. Hum Brain Mapp 2008;29:839-47

27. Greicius MD, Flores BH, Menon V, et al. Resting-state functional connectivity in major depression: abnormally increased contributions from subgenual cingulate cortex and thalamus. Biol Psychiatry 2007;62:429-37

28. Van Dijk KR, Hedden T, Venkataraman A, et al. Intrinsic functional connectivity as a tool for human connectomics: theory, properties, and optimization. J Neurophysiol 2010;103:297-321

29. Fox MD, Raichle ME. Spontaneous fluctuations in brain activity observed with functional magnetic resonance imaging. Nat Rev Neurosci 2007;8:700-11

30. Laufs H, Hamandi K, Salek-Haddadi A, et al. Temporal lobe interictal epileptic discharges affect cerebral activity in "default mode" brain regions. Hum Brain Mapp 2007;28:1023-32

31. Mankinen K, Jalovaara P, Paakki JJ, et al. Connectivity disruptions in resting-state functional brain networks in children with temporal lobe epilepsy. Epilepsy Res 2012;100:168-78

32. Supekar K, Uddin LQ, Prater K, et al. Development of functional and structural connectivity within the default mode network in young children. Neuroimage 2010;52:290-301

33. Luo C, Li Q, Lai Y, et al. Altered functional connectivity in default mode network in absence epilepsy: a resting-state fMRI study. Hum Brain Mapp 2011;32:438-49 